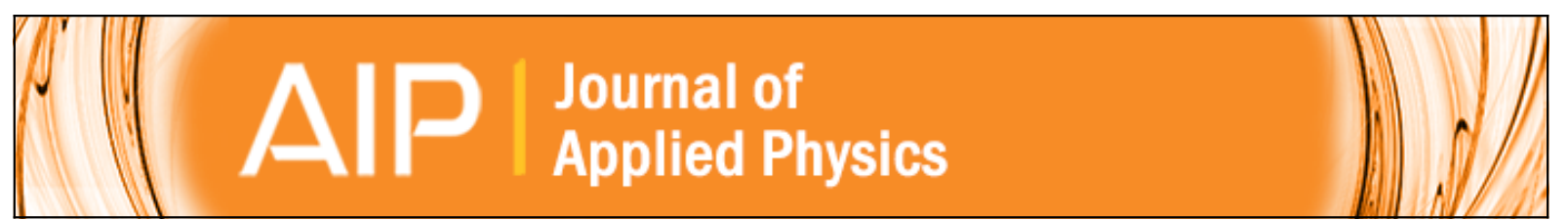

\title{
The dynamic behavior of thin-film ionic transition metal complex-based light-emitting electrochemical cells
}

Sebastian B. Meier, David Hartmann, Albrecht Winnacker, and Wiebke Sarfert

Citation: Journal of Applied Physics 116, 104504 (2014); doi: 10.1063/1.4895060

View online: http://dx.doi.org/10.1063/1.4895060

View Table of Contents: http://scitation.aip.org/content/aip/journal/jap/116/10?ver=pdfcov

Published by the AIP Publishing

\section{Articles you may be interested in}

Optically pumped amplified spontaneous emission in an ionic liquid-based polymer light-emitting electrochemical cell

Appl. Phys. Lett. 100, 263301 (2012); 10.1063/1.4730948

Encapsulating light-emitting electrochemical cells for improved performance

Appl. Phys. Lett. 100, 193508 (2012); 10.1063/1.4714696

Light-emitting electrochemical cells using polymeric ionic liquid/polyfluorene blends as luminescent material Appl. Phys. Lett. 96, 043308 (2010); 10.1063/1.3279155

Efficient solid-state host-guest light-emitting electrochemical cells based on cationic transition metal complexes Appl. Phys. Lett. 89, 261118 (2006); 10.1063/1.2425008

Steady state model for polymer light-emitting electrochemical cells

J. Appl. Phys. 81, 2869 (1997); 10.1063/1.363966

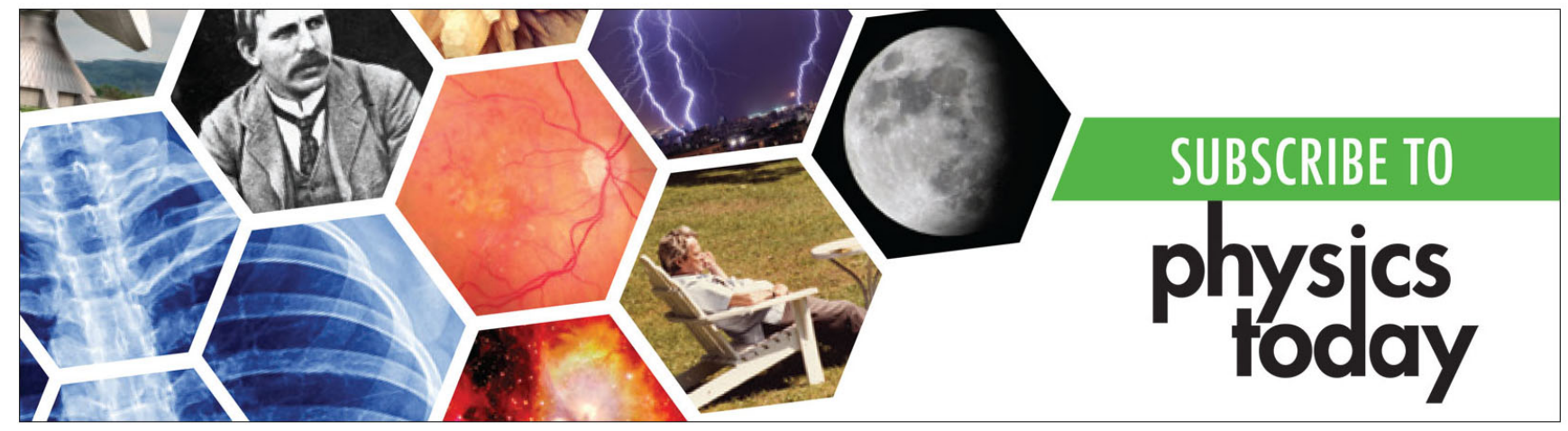




\title{
The dynamic behavior of thin-film ionic transition metal complex-based light-emitting electrochemical cells
}

\author{
Sebastian B. Meier, ${ }^{1,2, a), b)}$ David Hartmann, ${ }^{2}$ Albrecht Winnacker, ${ }^{1}$ and Wiebke Sarfert $\left.{ }^{2, b}\right)$ \\ ${ }^{1}$ Department of Materials Science VI: Materials for Electronics and Energy Technology, \\ Friedrich-Alexander-University of Erlangen-Nuremberg, 91058 Erlangen, Germany \\ ${ }^{2}$ Siemens AG, Corporate Technology, CT RTC MAT IEC-DE, 91058 Erlangen, Germany
}

(Received 4 August 2014; accepted 27 August 2014; published online 9 September 2014)

\begin{abstract}
Light-emitting electrochemical cells (LECs) have received increasing attention during recent years due to their simple architecture, based on solely air-stabile materials, and ease of manufacture in ambient atmosphere, using solution-based technologies. The LEC's active layer offers semiconducting, luminescent as well as ionic functionality resulting in device physical processes fundamentally different as compared with organic light-emitting diodes. During operation, electrical double layers (EDLs) form at the electrode interfaces as a consequence of ion accumulation and electrochemical doping sets in leading to the in situ development of a light-emitting p-i-n junction. In this paper, we comment on the use of impedance spectroscopy in combination with complex nonlinear squares fitting to derive key information about the latter events in thin-film ionic transition metal complex-based light-emitting electrochemical cells based on the model compound bis-2-phenylpyridine 6-phenyl-2,2'-bipyridine iridium(III) hexafluoridophosphate ([Ir(ppy $\left.\left.)_{2}\left(\mathrm{pbpy}_{2}\right)\right]\left[\mathrm{PF}_{6}\right]\right)$. At operating voltages below the bandgap potential of the ionic complex used, we obtain the dielectric constant of the active layer, the conductivity of mobile ions, the transference numbers of electrons and ions, and the thickness of the EDLs, whereas the transient thickness of the p-i-n junction is determined at voltages above the bandgap potential. Most importantly, we find that charge transport is dominated by the ions when carrier injection from the electrodes is prohibited, that ion movement is limited by the presence of transverse internal interfaces and that the width of the intrinsic region constitutes almost $60 \%$ of the total active layer thickness in steady state at a low operating voltage. (C) 2014 AIP Publishing LLC. [http://dx.doi.org/10.1063/1.4895060]
\end{abstract}

\section{INTRODUCTION}

Since the demonstration of the first inorganic lightemitting diode (LED) in $1962,{ }^{1}$ the rapid advancement in the field made these devices more and more an ubiquitous part of our everyday life. Nowadays, LEDs feature prominently in optical communications, lasing, display applications as well as in the lighting market, among other things owing to their high energy efficiency. ${ }^{2,3}$ In recent years, organic light-emitting diodes (OLEDs), the organic analogues to LEDs, have emerged as a promising alternative and have started to penetrate the market, especially as a technology for cell phone displays, but gradually also for TV screens and solid-state lighting. ${ }^{4-6}$ This is mainly attributed to their unique features of being surface light sources, selfluminous, flat, light-weight, and compatible with flexible substrates enabling new shapes and designs. Highly efficient state-of-the-art OLEDs typically feature a multilayer architecture comprising several layers with different functionalities (charge injection, charge transport, and radiative recombination) which are prepared via evaporation under high-vacuum and surrounding inert atmosphere. ${ }^{7,8}$ This makes the manufacturing process of OLEDs cost-intensive, especially with respect to larger areas, and therefore hinders

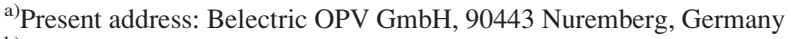

b) Authors to who correspondence should be addressed. Electronic addresses: sebastian.meier@belectric.com and wiebke.sarfert@siemens.com
}

a widespread penetration of the technology into the display and lighting market so far.

A promising approach is to reduce the complexity of the device by using materials that efficiently work in a singlelayer architecture and which are additionally solutionprocessible. A special kind of organic light-emitting device, referred to as light-emitting electrochemical cell (LEC), takes advantage of materials which fulfill both of the desired requirements. LECs are solid-state mixed ionic/electronic conductors comprising just a luminescent layer sandwiched in-between two electrodes. ${ }^{9-15}$ They consist either of a blend of a fluorescent, semiconducting polymer, an ionic conductor, and a salt and are termed polymer LECs (pLECs), ${ }^{9-12}$ or of a phosphorescent ionic transition metal complex (iTMC) usually admixed with an ionic liquid (IL) and are referred to as iTMC-LECs. ${ }^{13-15}$ The interplay between ionic and electronic charges in LECs leads to device processes that are fundamentally different from OLEDs. As a first step, the operation of LECs involves the accumulation of ions at the electrode interfaces in response to an applied electrical field. These thin sheets of uncompensated ionic charges, referred to as electric double layers (EDLs), facilitate the injection of electronic charge carriers into the active layer. ${ }^{16-19}$ Subsequent electrochemical doping of the luminescent, semiconducting material used, manifested via its reduction and oxidation by injected electrons and holes and the simultaneous compensation of the generated charge by ions with opposite sign, gives rise to the in situ formation of highly 
conductive $\mathrm{p}$ - and n-type regions adjacent to the anode and cathode, respectively, which grow towards each other to finally establish a light-emitting p-i-n junction, where charge carriers recombine radiatively., ${ }^{9,20,21}$ The peculiar functional principle of LECs is responsible for their beneficial properties, like low-voltage operation and bipolar electroluminescence almost irrespective of the work-function of the electrode materials used, ${ }^{9,10,22,23}$ but has been the subject of intense debates ever since their discovery by Pei et al. in $1995{ }^{24-26}$ Recent studies, however, have unequivocally confirmed the working mechanism. ${ }^{27,28}$

Most of the experiments performed to shed light on the functional principle of LECs were conducted on devices with a planar geometry featuring active layer thicknesses up to the centimeter scale. ${ }^{29-31}$ The device design is enabled by the formation of the highly conductive, doped regions during operation and allows for the use of analysis techniques like optical imaging via fluorescence microscopy, ${ }^{21,27,29-33}$ scanning Kelvin probe microscopy (SKPM), ${ }^{20,21,27,32,34}$ and optical beam induced current (OBIC) measurements. ${ }^{33,35,36}$ However, LECs that are relevant for practical use comprise a stacked architecture with an active layer of only a few hundreds of nanometers. This huge discrepancy in thickness raises the question whether one can directly translate the working mechanism from planar to sandwiched LECs. Especially in recent years, the physical processes in stacked devices were studied by different electrical and optical measurement techniques ${ }^{37-40}$ and among them impedance spectroscopy (IS) turned out to be a promising approach. As this method uses a time-dependent signal, it is possible to look separately at fast (electronic) and slow (ionic) processes in LECs which makes IS an excellent means to disentangle both charge transport mechanisms in operational devices. So far most of the IS work was focused on pLECs. ${ }^{41-48}$ While in the early days, IS was used to probe the steady- or quasisteady state condition of these devices, ${ }^{41-44}$ lately also their transient properties were investigated. ${ }^{45-48}$ Two very recent studies mediate a very comprehensive picture of the physics of pLEC devices, including the extraction of key information, like the dielectric constant of the active layer, the conductivity of mobile ions, the thickness of the EDLs, and the width of the intrinsic region as a function of time and voltage. ${ }^{47,48}$ The utilization of IS in case of iTMC-LECs, especially with respect to the evaluation of physical processes, is, however, rather scarce. It was applied to study degradation in these devices ${ }^{49,50}$ to make statements about the impact of ion mobilities, ${ }^{51}$ to prove electrochemical junction formation and to highlight influencing variables on the junction width, and to estimate the transient thickness of the p-i-n junction during operation. ${ }^{38,52}$ One of the latest publications in the field of LECs used IS to demonstrate that pLECs and iTMCLECs are essentially behaving as one class of device. ${ }^{53}$

In this paper, the results from an impedance spectroscopy study on stacked iTMC-LECs based on the ionic iridium(III) model compound bis-2-phenylpyridine 6-phenyl-2,2'-bipyridine iridium(III) hexafluoridophosphate $\left(\left[\operatorname{Ir}(\mathrm{ppy})_{2}(\mathrm{pbpy})\right]\left[\mathrm{PF}_{6}\right]\right)$ are highlighted. The evaluation of the corresponding data is additionally supported by means of complex nonlinear least squares (CNLS) fitting to finally establish a comprehensive picture about the physics and dynamics of these kind of devices. We find that charge transport in the investigated devices is dominated by the ions when carrier injection from the electrodes is prohibited and that ion movement is in general limited by the presence of transverse internal interfaces. The mobility of $\mathrm{PF}_{6}{ }^{-}$anions is calculated to be around $10^{-12} \mathrm{~cm}^{2} / \mathrm{Vs}$ and the EDL thickness to lie in between 6 and $7 \mathrm{~nm}$. We also operated the devices at a low voltage of $3 \mathrm{~V}$, slightly higher than the bandgap potential of the ionic complex used, and show that the width of the intrinsic region constitutes almost $60 \%$ of the total active layer thickness in steady state which is obviously the cause for the low current and luminance observed under these driving conditions.

\section{EXPERIMENTAL SECTION}

\section{A. Device fabrication}

$\left[\operatorname{Ir}(\mathrm{ppy})_{2}(\mathrm{pbpy})\right]\left[\mathrm{PF}_{6}\right]$ was synthesized similarly according to methods described previously. ${ }^{54,55}$ Poly(3,4-ethylenedioxythiophene) poly(styrenesulfonate) (PEDOT:PSS) aqueous dispersion (Clevios ${ }^{\mathrm{TM}}$ P VP CH8000) was purchased from Heraeus. Acetonitrile (anhydrous, 99.8\%) and the IL 1-butyl-3-methylimidazolium hexafluoridophosphate $[\mathrm{BMIM}]\left[\mathrm{PF}_{6}\right]$ (purum, $\geq 97.0 \%$ ) were obtained from Sigma Aldrich and used as-received. Indium tin oxide (ITO)-coated glass substrates (ShinAn SNP) were patterned using conventional photolithography.

Sandwich-type solid-state light-emitting electrochemical cells were fabricated via spin-coating under ambient conditions. For that the ITO substrates were extensively cleaned using sonication in detergent bath and distilled water as well as using subsequent oxygen plasma treatment. Prior to the coating of the active (emissive) layer, a $100 \mathrm{~nm}$ film of PEDOT:PSS was deposited $(1500 \mathrm{rpm}, 60 \mathrm{~s})$ to planarize the ITO anode increasing the yield and reproducibility of working devices. A stock solution was prepared dissolving $5 \%$ by weight of the iridium complex and the IL in a molar ratio of 3:1 in acetonitrile. The IL was added to decrease the turn-on time of the devices. ${ }^{56}$ The stock solution was filtered using a $0.1 \mu \mathrm{m}$ polytetrafluoroethylene (PTFE)-filter and spin-coated on top of the PEDOT:PSS layer $(750 \mathrm{rpm}, 60 \mathrm{~s})$. Afterwards the substrates were transferred to an argon filled glovebox $\left(\mathrm{O}_{2}\right.$ and $\mathrm{H}_{2} \mathrm{O}<1 \mathrm{ppm}$ ) and annealed on a hotplate at $100^{\circ} \mathrm{C}$ for $1 \mathrm{~h}$ resulting in a final active layer thickness of $100 \mathrm{~nm}$. Finally, a $150 \mathrm{~nm}$ thick aluminum (Al) cathode was thermally evaporated on top under high vacuum $\left(<1 \times 10^{-6}\right.$ mbar) using a shadow mask, defining 8 pixels per substrate, each having an active lighting area of $4 \mathrm{~mm}^{2}$. Thicknesses of the films were determined on reference samples using a KLA Tencor P. 15 profilometer.

To investigate the devices under ambient conditions, they were encapsulated under inert atmosphere by a cavity glass capping (Shanghai Amerina Optoelectronic) equipped with a silica getter to protect the pixel areas against penetrating oxygen and water. The capping was directly glued to the substrate without prior removal of the organic layers using a self-made UV-curable epoxy adhesive which was coated locally onto the margin of the capping. To test the functionality of the encapsulation, control devices were processed using a metallic calcium layer as a sensor. As the calcium 
sensor stayed metallic during the long-term study of several hundreds of hours, a possible reaction with water and oxygen to $\mathrm{CaO}$ or $\mathrm{Ca}(\mathrm{OH})_{2}$ which would have changed the optical appearance and the resistivity of the transparent film, could be excluded.

\section{B. Impedance spectroscopy}

IS was performed to obtain key information of iTMCLECs based on the ionic complex $\left[\operatorname{Ir}(\mathrm{ppy})_{2}(\mathrm{pbpy})\right]\left[\mathrm{PF}_{6}\right]$, like the conductivity and mobility, respectively, of mobile ions, the thickness of the EDLs, and the width of the intrinsic region of the $\mathrm{p}-\mathrm{i}-\mathrm{n}$ junction during operation.

In IS, a small alternating voltage $\mathrm{v}_{\mathrm{ac}}=\mathrm{v}_{0} \sin (\omega \mathrm{t})$ is applied between the two device electrodes and the resulting current response $\mathrm{i}_{\mathrm{ac}}=\mathrm{i}_{0} \sin (\omega \mathrm{t}+\varphi)$ is detected with $\mathrm{v}_{0}$ and $\mathrm{i}_{0}$ representing the corresponding amplitudes, $\omega$ the angular frequency ( $\omega=2 \pi \mathrm{f}$, where $\mathrm{f}$ is the frequency), and $\varphi$ the phase difference between the voltage and the current. The parameters defined by IS depend on the current response $i_{a c}$ and can be decomposed in a real part and an imaginary part according to

$$
Y=\frac{1}{Z}=\frac{i_{a c}}{v_{a c}}=G(\omega)+i \omega C(\omega),
$$

where $\mathrm{Y}, \mathrm{Z}, \mathrm{G}$, and $\mathrm{C}$ represent the admittance, the impedance, the conductance, and the capacitance of the system of interest, respectively.

The impedance analysis was executed at a constant temperature of $20^{\circ} \mathrm{C}$ (controlled by the use of a Peltier element) either with a Schlumberger SI 1260 Impedance-Gain Phase Analyzer in the frequency range from $1 \mathrm{MHz}$ to $10^{-3} \mathrm{~Hz}$ or with an Agilent 4294 A Precision Impedance Analyzer in the frequency range from $1 \mathrm{MHz}$ to $40 \mathrm{~Hz}$. As variations in the modulation voltage amplitude $\mathrm{v}_{\mathrm{ac}}$ in the range of $10-100 \mathrm{mV}$ showed minor scattering of the measuring points in the low frequency part of the impedance data for small values of $\mathrm{v}_{\mathrm{ac}}$ $(\leq 50 \mathrm{mV})$, an AC voltage of $100 \mathrm{mV}$ was chosen. The impedance spectra were recorded either on pristine LECs without an offset voltage $\mathrm{v}_{\mathrm{dc}}$ applied or during the operation of the devices at a defined constant $\mathrm{v}_{\mathrm{dc}}$ of $3 \mathrm{~V}$ superimposed on $\mathrm{v}_{\mathrm{ac}}$. The electrical control and the data acquisition were accomplished by a custom-made LabVIEW program.

\section{Fitting of impedance data}

Using a CNLS procedure, the IS data were fitted to the response of an equivalent circuit yielding estimates of the parameters of the model. CNLS fitting was performed by means of the program LEVM/LEVMW by James Ross Macdonald which is available free of charge via the internet (www.jrossmacdonald.com; present version 8.12). For the fitting of the impedance data, the use of constant phase elements (CPEs) was necessary. A CPE is an equivalent electrical circuit component that models the behavior of an imperfect capacitor. The impedance of the CPE is defined as

$$
Z_{C P E}=\frac{1}{Q(i \omega)^{\alpha}},
$$

where $Q$ represents the numerical value of $1 /|\mathrm{Z}|$ at $\omega=1 \mathrm{rad} / \mathrm{s}$ and $\alpha$ the phase of the element. Both $\mathrm{Q}$ and $\alpha(0 \leq \alpha \leq 1)$ are frequency-independent parameters. The interpretation of $\mathrm{Q}$ depends on $\alpha$ and cannot be directly associated with a meaningful physical value. However, when $\alpha=1$, the CPE acts as a pure capacitor and when $\alpha=0$, it acts as a pure resistor. For other $\alpha$ values, it is possible to extract an effective device capacitance $\mathrm{C}_{\text {eff }}$, via an approach developed by Brug et al. ${ }^{57}$ and Hirschorn et al. ${ }^{58}$ using the equation

$$
C_{e f f}=Q^{1 / \alpha} R^{(1-\alpha) / \alpha}
$$

where $\mathrm{R}$ represents a series resistance in a blocking-electrode scenario and a parallel resistance in a non-blocking-electrode scenario. Possible explanations for the appearance of a CPE include the surface roughness of the electrodes, inhomogeneous reaction rates at the surface, a varying thickness and/or composition, and a non-uniform current distribution. ${ }^{59}$

\section{RESULTS AND DISCUSSION}

\section{A. Analysis of the iTMC-LEC in the initial state}

The frequency-dependent impedance and phase angle of a pristine iTMC-LEC recorded at a bias voltage of $0 \mathrm{~V}$ are illustrated in Figure 1(a), whereas the corresponding capacitance and conductance are depicted in Figure 1(b). In accordance with the results of van Reenen et al. obtained for a stacked pLEC, ${ }^{48}$ a conductance plateau $\left(6.97 \times 10^{-7} \mathrm{~S}\right)$ is present at longer timescales $(10-1000 \mathrm{~Hz})$ which can be attributed to ion transport. Moreover, in the frequency range between $100 \mathrm{~Hz}$ and $10 \mathrm{kHz}$, a capacitance plateau $(1.81 \mathrm{nF})$ is observed which can be ascribed to the geometrical capacitance $\mathrm{C}_{\mathrm{geo}}$ of the device. By employing the equation

$$
C=\frac{\epsilon_{0} \epsilon_{r} A}{d}
$$
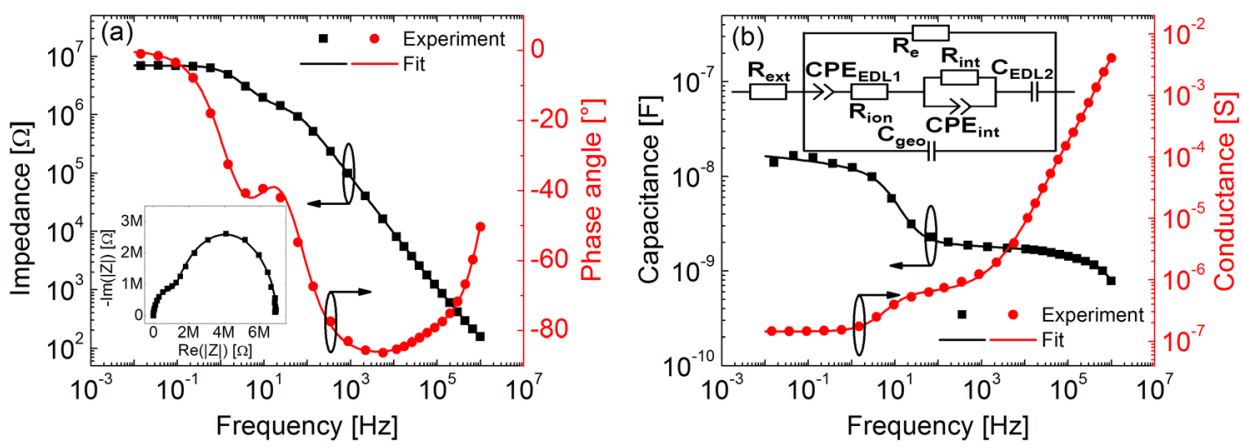

FIG. 1. (a) Frequency-dependent impedance and phase angle and (b) corresponding capacitance and conductance of an iTMC-LEC recorded at a bias voltage of $0 \mathrm{~V}$. The inset in (a) illustrates the respective Cole-Cole plot. The solid lines represent the best fit of the parameters extracted from the equivalent circuit model depicted as an inset in (b) to the measured data. 
with $\varepsilon_{0}$ being the permittivity of free space (8.85 $\times 10^{-12} \mathrm{~F} / \mathrm{m}$ ), and using geometric data of the LEC device (area $\mathrm{A}$ and thickness of the active layer $\mathrm{d}$ ), a dielectric constant $\varepsilon_{\mathrm{r}}$ of 5.11 is computed. When approaching lower frequencies, an increase in the capacitance at around $40 \mathrm{~Hz}$ is evident passing over to a second plateau $(14.69 \mathrm{nF})$. This finding can be correlated to the formation of EDLs which screen the electrical field in the bulk of the device. Accordingly, the conductance starts to decrease at the same time at which the EDLs start to form to finally pass over to a second conductance plateau at frequencies $<1 \mathrm{~Hz}\left(1.44 \times 10^{-7} \mathrm{~S}\right)$ which can be assigned to electronic leakage. Besides the similarities in the general trend of the impedance spectra obtained in this study and for the pLECs probed by van Reenen et al. ${ }^{48}$ the spectra of the latter devices are shifted by at least two orders of magnitude to higher frequencies, meaning that iTMCbased cells respond much slower to an electrical signal than pLECs do. This rationalizes the fact that polymer-based devices reveal turn-on times significantly shorter (seconds) compared with their ionic, small-molecular counterparts (hours).

The results highlighted in Figure 1(b) can be used to obtain information about the thickness of the EDLs and the ionic conductivity. Assuming that the capacitance of both EDLs $\left(\mathrm{C}_{\mathrm{EDL}}\right)$ is similar (i.e., $29.38 \mathrm{nF}$ in total $)^{60}$ and that the layer in between the sheet of ions and the electrodes has the same dielectric constant as the rest of the film, the thickness of the EDLs $\mathrm{d}_{\mathrm{EDL}}$ can be approximated as

$$
d_{E D L} C_{E D L}=d C_{g e o}
$$

must hold. ${ }^{48}$ Using the experimental data, an EDL thickness of $6.16 \mathrm{~nm}$ can be calculated which is in good agreement with recent literature results on pLECs. ${ }^{47,48}$ Such a small width allows for easy tunneling injection of electronic charge carriers into the active layer during operation at voltages above the bandgap potential of the iTMC used. On the other hand, the conductance plateau at intermediate frequencies can be used to determine the conductivity $\sigma$ of the ions in the active layer according to equation

$$
\sigma=\frac{G d}{A},
$$

where a value of $1.74 \times 10^{-10} \mathrm{~S} / \mathrm{cm}$ is obtained. Surprisingly, this is at least on order of magnitude higher as found for ruthenium(II)-based LECs, ${ }^{51}$ although the turn-on times of the latter devices are much faster (seconds) compared with the iridium(III) one under study (hours). This may be rationalized by the fact that p-doping initially dominates in the iridium(III)based iTMC-LEC and hence the onset of light emission occurs in close proximity to the metal electrode. ${ }^{21}$ As metals are known to heavily quench the luminescence of organic compounds, the emission zone has to move away first before light emission can be detected, which is why the turn-on time is longer.

Recalling that the mobility of the bulky iridium(III) cation is negligible ${ }^{21}$ and that the dimension of the IL cation $\left([\mathrm{BMIM}]^{+}\right.$) may result in a low mobility as well, the ion conductivity is ascribable to the small $\mathrm{PF}_{6}{ }^{-}$anions. This aspect allows for the calculation of their mobility. Considering a total anion density of $8.25 \times 10^{20} \mathrm{~cm}^{-3}$, which can be estimated taking account of the ratio between iridium(III) complex and IL and of the dimensions of the device active layer and by assuming an active layer density of $1 \mathrm{~g} / \mathrm{cm}^{3}$, an anion mobility of $1.32 \times 10^{-12} \mathrm{~cm}^{2} / \mathrm{Vs}$ is determined. Making use of Einstein's relation, this corresponds to an anion diffusion constant of $3.39 \times 10^{-14} \mathrm{~cm}^{2} / \mathrm{s}$.

Finally, the contributions of the electronic and ionic species to the transport of charge can be calculated which is referred to as the transference number of the respective species. ${ }^{61}$ Using the values for the ionic and electronic conductance and the equations illustrated in Ref. 61, transference numbers for ions $\left(t_{i}\right)$ and electrons $\left(t_{e}\right)$ of 0.83 and 0.17 are obtained implying that charge transport in the iTMC-LEC is dominated by the ions at operating voltages below the bandgap potential of the ionic iridium(III) complex, i.e., when charge carrier injection from the electrodes is prohibited.

To corroborate the previous findings, CNLS data analysis was executed to fit the experimental data to a physically motivated equivalent circuit model. ${ }^{59,62}$ The equivalent circuit that has been used is depicted as an inset in Figure 1(b) and consists of three parallel branches. $\mathrm{R}_{\mathrm{e}}$ represents the electrical resistance of the active layer, including the injection resistances. $\mathrm{C}_{\mathrm{geo}}$ illustrates the geometrical capacitance of the two electrodes contacting the active layer. The third path reflects the existence of ions within the active layer and comprises a series assembly of various elements. The constant phase element $\mathrm{CPE}_{\mathrm{EDL} 1}$ and the capacitor $\mathrm{C}_{\mathrm{EDL} 2}$ denote the two EDLs at the electrode/active layer interfaces, while the resistor $\mathrm{R}_{\text {ion }}$ represents the bulk ionic resistance of the active layer. Up to this point, the equivalent circuit is virtually identical to the one used by Munar et al. to model the impedance data of a pLEC. ${ }^{47}$ However, Munar's approach did not lead to a good fitting result in case of the herein investigated iTMC-LEC, for which reason it was modified by an additional parallel resistor/CPE array $\mathrm{R}_{\text {int }} / \mathrm{CPE}_{\text {int }}$. According to Huggins, this element reflects an additional impedance due to transverse internal interfaces, arising, for example, from grain or interphase boundaries in polycrystalline materials. ${ }^{61}$ It was reported that alternating laminar domains of cations and anions, respectively, emerge in the crystal structure of $\left[\operatorname{Ir}(\mathrm{ppy})_{2}(\mathrm{pbpy})\right]\left[\mathrm{PF}_{6}\right] .{ }^{54}$ If this architecture is maintained in thin films containing small amounts of IL, this may rationalize the additional impedance as ionic transport across the domains is hampered as opposed to transport inside the domains. Otherwise, one may also assume nanoscale crystallization as a possible source for the additional impedance. Finally, the parallel circuit assembly is connected in series with another resistance $\left(\mathrm{R}_{\mathrm{ext}}\right)$, representing all external resistances, including those of the ITO and Al electrodes and the measurement cables. It is important to mention that in order to obtain a good fit of the model to the experimental data, the partial use of CPEs instead of pure capacitances is essential. The need for CPE elements is generally attributed to the existence of surface and/or bulk heterogeneities or to continuously distributed time constants for chargetransfer reactions. ${ }^{58,59}$ The fact that no CPE was employed to model the second EDL is accounted for by restrictions in the fitting software used. It is, however, not certain that a CPE instead of a capacitor will result in a better fit to the 
TABLE I. Equivalent circuit data for an iTMC-LEC at a bias voltage of $0 \mathrm{~V}$.

\begin{tabular}{lccccc}
\hline \hline $\mathrm{R}_{\text {ext }}[\Omega]^{\mathrm{a}}$ & $\mathrm{R}_{\mathrm{e}}[\Omega]^{\mathrm{b}}$ & $\mathrm{C}_{\text {geo }}[\mathrm{F}]^{\mathrm{c}}$ & $\mathrm{Q}_{\mathrm{EDL} 1}{ }^{\mathrm{d}}$ & $\alpha_{\mathrm{EDL} 1}{ }^{\mathrm{e}}$ & $\mathrm{C}_{\mathrm{EDL} 1}[\mathrm{~F}]^{\mathrm{f}}$ \\
\hline \hline 99 & $6.98 \times 10^{6}$ & $1.32 \times 10^{-9}$ & $3.95 \times 10^{-8}$ & 0.79 & $4.03 \times 10^{-9}$ \\
\hline \hline $\mathrm{R}_{\text {ion }}[\Omega]^{\mathrm{g}}$ & $\mathrm{C}_{\mathrm{EDL} 2}[\mathrm{~F}]^{\mathrm{h}}$ & $\mathrm{R}_{\text {int }}[\Omega]^{\mathrm{i}}$ & $\mathrm{Q}_{\text {int }}{ }^{\mathrm{j}}$ & $\alpha_{\text {int }}{ }^{\mathrm{k}}$ & $\mathrm{C}_{\text {int }}[\mathrm{F}]^{1}$ \\
\hline $4.30 \times 10^{3}$ & $1.91 \times 10^{-8}$ & $1.63 \times 10^{6}$ & $1.34 \times 10^{-9}$ & 0.90 & $7.06 \times 10^{-10}$ \\
\hline \hline
\end{tabular}

${ }^{\mathrm{a}}$ External resistance (electrodes, cables, etc.).

${ }^{\mathrm{b}}$ Electrical resistance.

${ }^{\mathrm{c}}$ Geometrical capacitance.

${ }^{\mathrm{d}}$ Numerical value of $1 /|\mathrm{Z}|$ at $\omega=1 \mathrm{rad} / \mathrm{s}$ of the CPE representing EDL 1 .

${ }^{\mathrm{e}}$ Phase of the CPE representing EDL 1.

${ }^{\mathrm{f}}$ Capacitance of electrical double layer 1 .

g Ionic resistance.

${ }^{\mathrm{h}}$ Capacitance of electrical double layer 2.

${ }^{\mathrm{i}}$ Interfacial resistance.

${ }^{\mathrm{j}}$ Numerical value of $1 /|\mathrm{Z}|$ at $\omega=1 \mathrm{rad} / \mathrm{s}$ of the CPE representing interfacial charge transfer.

${ }^{\mathrm{k}}$ Phase of the CPE representing interfacial charge transfer.

${ }^{1}$ Interfacial capacitance.

experimental results. One may envision that a PEDOT:PSS layer is present in the device to smoothen the typical uneven surface structure of ITO for which reason a simple capacitor may be adequate to model the anodic interface.

The solid lines in Figure 1 represent the fitting results of the equivalent circuit model to the experimental data. The very good agreement between the data and the modeling results confirms that the selected equivalent circuit is an appropriate choice. The individual variables obtained from the fit are depicted in Table I, whereas the effective capacitances $\mathrm{C}_{\text {eff }}$ of the CPEs have been calculated according to Eq. (3). The quantities enable the calculation of specific device parameters of the current iTMC-LEC. By employing Eq. (4) and using geometric data of the LEC device, a dielectric constant of 3.72 is computed. It is somewhat lower than the experimental finding highlighted before which is due to a slightly different geometrical capacitance. When approximating the structure of the EDLs with that of a planar capacitor, their widths can be calculated according to Eq. (4) as well by applying the calculated dielectric constant. Thicknesses of 6.89 and $32.64 \mathrm{~nm}$ are derived, where the latter is rather high with respect to the total active layer thickness of $100 \mathrm{~nm}$. This is due to a low $\alpha$ value of the respective CPE. A similar result was obtained by Munar et al. and was ascribed to a rather inhomogeneous electrode interface. ${ }^{47}$ Even if the computed thickness of the second EDL seems quite unrealistic, it is worth mentioning that the occurrence of large EDLs may be generally possible as concluded from the outcomes obtained in case of planar iTMC-LECs. ${ }^{21}$ In contrast, the width of the thinner EDL is in excellent agreement with the experimental findings as illustrated before. This holds also for the ionic and electronic conductance derived from the respective resistances. Accordingly, similar experimental and modeling results are also achieved for the transference numbers of ions and electrons and the conductivity, mobility, and diffusion constant of anions. A summary of the typical device parameters of the $\left[\operatorname{Ir}(\mathrm{ppy})_{2}\right.$ (pbpy)][$\left[\mathrm{PF}_{6}\right]$-based LEC, extracted from the experimental findings and from equivalent circuit modeling, is depicted in Table II. Finally, one can conclude that the large discrepancy between $\mathrm{R}_{\text {ion }}$ and $\mathrm{R}_{\text {int }}$ (see Table $\mathrm{I}$ ) implies that ion transport in the present device is limited by internal interfaces, either due the occurrence of nanoscale crystallization or in consequence of the formation of ion domains as outlined above.

\section{B. Transient behavior of the ITMC-LEC in operation}

The IS investigations at a bias voltage of $0 \mathrm{~V}$ provided already a multitude of valuable information about structural parameters of $\left[\operatorname{Ir}(\mathrm{ppy})_{2}(\mathrm{pbpy})\right]\left[\mathrm{PF}_{6}\right]$-based LECs. As a next step, it is also important to study the transient impedance response when a voltage higher than the bandgap potential of the iridium(III) complex (2.6 V) is applied. This allows for the analysis of the electrochemical doping process and for the calculation of the junction width of the evolving $\mathrm{p}-\mathrm{i}-\mathrm{n}$ structure as a function of operating time. It is to be noticed that the thickness of the intrinsic region was found to be strongly dependent on the operating voltage. ${ }^{41,47,52}$

TABLE II. Typical device parameters of the iTMC-LEC extracted from experimental results and equivalent circuit modeling.

\begin{tabular}{lccccc}
\hline \hline & $\varepsilon_{\mathrm{r}}^{\mathrm{a}}$ & $\mathrm{d}_{\mathrm{EDL} 1}[\mathrm{~nm}]^{\mathrm{b}}$ & $\mathrm{d}_{\mathrm{EDL} 2}[\mathrm{~nm}]^{\mathrm{c}}$ & $\mathrm{t}_{\text {ion }}{ }^{\mathrm{d}}$ & 0.83 \\
\hline Experiment & 5.11 & 6.16 & 6.16 & 0.81 & 0.17 \\
Model & 3.72 & 32.64 & 6.89 & $\mathrm{t}_{\mathrm{e}}{ }^{\mathrm{e}}$ \\
\hline \hline & $\mathrm{G}_{\mathrm{ion}}[\mathrm{S}]^{\mathrm{f}}$ & $\mathrm{G}_{\mathrm{e}}[\mathrm{S}]^{\mathrm{g}}$ & $\sigma_{\text {anion }}\left[\mathrm{S} / \mathrm{cm}^{\mathrm{h}}\right.$ & $\mu_{\text {anion }}\left[\mathrm{cm}^{2} / \mathrm{Vs}^{\mathrm{i}}\right.$ & $1.32 \times 10^{-12}$ \\
\hline Experiment & $6.97 \times 10^{-7}$ & $1.44 \times 10^{-7}$ & $1.74 \times 10^{-10}$ & $1.15 \times 10^{-12}$ & $3.39 \times 10^{-14}$ \\
Model & $6.09 \times 10^{-7}$ & $1.43 \times 10^{-7}$ & $1.52 \times 10^{-10}$ & $2.97 \times 10^{-14}$ \\
\hline \hline
\end{tabular}

${ }^{\mathrm{a}}$ Dielectric constant (relative permittivity).

${ }^{\mathrm{b}}$ Thickness of electrical double layer 1 .

${ }^{\mathrm{c}}$ Thickness of electrical double layer 2.

${ }^{\mathrm{d}}$ Transfer number of ions.

${ }^{\mathrm{e}}$ Transfer number of electrons.

${ }^{\mathrm{f}}$ Ionic conductance.

${ }^{g}$ Electrical conductance.

${ }^{\mathrm{h}}$ Anion conductivity.

${ }^{\mathrm{i}}$ Anion mobility.

${ }^{\mathrm{j}}$ Diffusion constant of anions. 


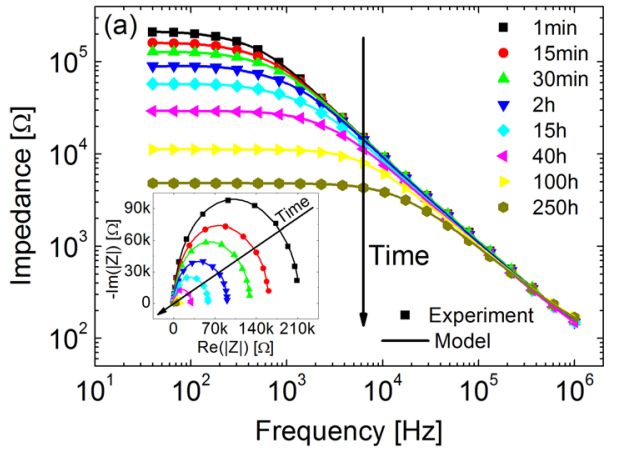

The transient impedance and phase angle of the iTMCLEC as a function of frequency recorded at a bias voltage of $3 \mathrm{~V}$ are illustrated in Figure 2. Applying the bias to the device leads to a continuous decrease in the impedance with operating time (Figure 2(a)). Simultaneously, the minimum of the phase angle increases and shifts to higher frequencies (Figure 2(b)). The corresponding Cole-Cole plots, depicted as an inset in Figure 2(a), appear to consist of single semicircles with radii that decrease monotonously with increasing operating time implying an ongoing reduction of the device resistance. This behavior can be explained by the formation and growth of highly conductive regions starting from the electrode interfaces that decrease the width of a less conductive, intrinsic (undoped) region sandwiched in between.

To estimate the thickness of the intrinsic region as a function of driving time, CNLS equivalent circuit modeling was performed. According to Munar et al., an LEC under operation can be illustrated by two resistors which represent the doped regions and a resistor $\mathrm{R}_{\mathrm{i}}$ connected in parallel with a constant phase element $\mathrm{CPE}_{\mathrm{i}}$ to take into account the resistive and capacitive components of the intermediately positioned p-i-n junction. ${ }^{47}$ This approach, however, did not lead to a good fitting result in case of the herein investigated iTMC-LEC. Therefore, the equivalent circuit was modified and the resistors representing the doped regions were replaced by parallel resistor/CPE assemblies $\mathrm{R}_{\mathrm{n}, \mathrm{p}} / \mathrm{CPE}_{\mathrm{n}, \mathrm{p}}$ as shown in the inset of Figure 2(b). This may be necessary owing to a gradual change in the charge carrier density and conductivity throughout the doped regions as it was demonstrated to occur in pLECs. ${ }^{48}$ Charge carriers that enter regions of lower density/conductivity cause an accumulation of charges which is reflected in a capacitive contribution. As in the data fitting carried out before, the use of CPEs instead of pure capacitances is essential in order to obtain a good fit of the model to the experimental data.

The solid lines in Figure 2 represent the fitting results of the equivalent circuit model to the experimental data. The very good agreement between the data and the modeling results confirms that the selected equivalent circuit is an appropriate choice. The individual variables obtained from the fit which describe the intrinsic region of the iTMC-LEC are depicted in Table III. Again, the capacitance of the CPE has been calculated according to Eq. (3). It is obvious that the resistance of the undoped zone decreases markedly during operation, while its capacitance increases steadily which is also illustrated in Figure 3. By approximating the junction region by a parallel plate capacitor, the width of the intrinsic region $d_{i}$ can be estimated from the calculated capacitance, the experimentally determined dielectric constant, and the geometrical dimensions of the device. The results are enclosed in Table III and in Figure 3 together with the simultaneously recorded current density over time. In the early stage of operation, the calculated thickness of $103 \mathrm{~nm}$ agrees well with the nominal thickness of the device $(100 \mathrm{~nm})$. While the resistance of the undoped zone drops significantly during the first hour of operation, its capacitance and hence

TABLE III. Selected time-dependent device data of an iTMC-LEC at a bias voltage of $3 \mathrm{~V}$ derived from equivalent circuit modeling. In addition, also current and luminance data are listed.

\begin{tabular}{|c|c|c|c|c|c|c|c|}
\hline Time $[\mathrm{h}]$ & $\mathrm{R}_{\mathrm{i}}[\Omega]^{\mathrm{a}}$ & $\mathrm{Q}_{\mathrm{i}}^{\mathrm{b}}$ & $\alpha_{i}^{c}$ & $\mathrm{C}_{\mathrm{i}}[\mathrm{F}]^{\mathrm{d}}$ & $\mathrm{d}_{\mathrm{i}}[\mathrm{nm}]^{\mathrm{e}}$ & $\mathrm{j}\left[\mathrm{mA} / \mathrm{cm}^{2}\right]^{\mathrm{f}}$ & $\mathrm{L}\left[\mathrm{cd} / \mathrm{m}^{2}\right]^{\mathrm{g}}$ \\
\hline 0.02 & $1.79 \times 10^{5}$ & $3.14 \times 10^{-9}$ & 1.00 & $1.76 \times 10^{-9}$ & 103 & 0.02 & 0 \\
\hline 0.25 & $1.39 \times 10^{5}$ & $1.89 \times 10^{-9}$ & 0.99 & $1.76 \times 10^{-9}$ & 103 & 0.03 & 0 \\
\hline 0.5 & $1.12 \times 10^{5}$ & $1.94 \times 10^{-9}$ & 0.99 & $1.76 \times 10^{-9}$ & 103 & 0.05 & 0 \\
\hline 2 & $7.59 \times 10^{4}$ & $2.03 \times 10^{-9}$ & 0.99 & $1.81 \times 10^{-9}$ & 100 & 0.06 & 0 \\
\hline 15 & $4.67 \times 10^{4}$ & $2.91 \times 10^{-9}$ & 0.96 & $2.06 \times 10^{-9}$ & 88 & 0.11 & 0 \\
\hline 40 & $2.03 \times 10^{4}$ & $3.11 \times 10^{-9}$ & 0.97 & $2.41 \times 10^{-9}$ & 75 & 0.28 & 8 \\
\hline 100 & $9.08 \times 10^{3}$ & $2.93 \times 10^{-9}$ & 1.00 & $2.87 \times 10^{-9}$ & 63 & 0.93 & 46 \\
\hline 250 & $3.62 \times 10^{3}$ & $4.65 \times 10^{-9}$ & 0.97 & $3.14 \times 10^{-9}$ & 58 & 2.59 & 104 \\
\hline
\end{tabular}

${ }^{\mathrm{a}}$ Resistance of the intrinsic (undoped) region.

${ }^{\mathrm{b}}$ Numerical value of $1 /|\mathrm{Z}|$ at $\omega=1 \mathrm{rad} / \mathrm{s}$ of the CPE representing the intrinsic (undoped) region.

${ }^{\mathrm{c}} \mathrm{Phase}$ of the CPE representing the intrinsic (undoped) region.

${ }^{\mathrm{d}}$ Capacitance of the intrinsic (undoped) region.

${ }^{\mathrm{e}}$ Thickness of the intrinsic (undoped) region.

${ }^{\mathrm{f}}$ Current density.

${ }^{\mathrm{g}}$ Luminance. 


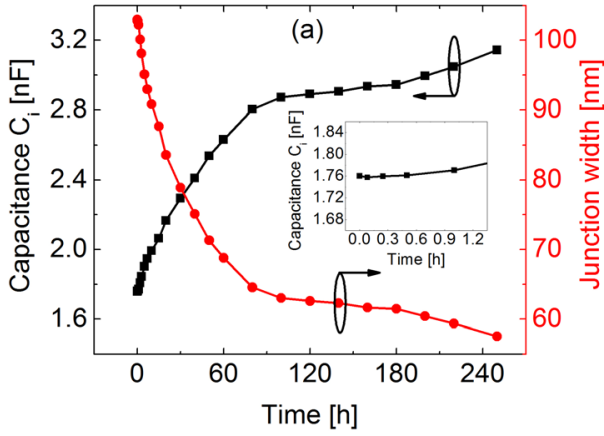

FIG. 3. (a) Time-dependent capacitance of the intrinsic region and corresponding junction width and (b) resistance of the intrinsic region and device current density of an iTMCLEC at a bias voltage of $3 \mathrm{~V}$. the junction width are almost constant (see Figure 3 insets). This may be explained by the slow formation of the EDLs which gradually reduce the injection resistance. The current increases only slightly as a result of the large bulk resistance. When the injection barriers are overcome, electrochemical doping sets in and conductive regions start to grow from the electrode interfaces. The width of the intrinsic region decreases continuously reflected by the rise in the capacitance, the ongoing decline in the resistance, and the increment in the current. After around $100 \mathrm{~h}$ of operation, the thickness of the undoped zone has reduced to $63 \mathrm{~nm}$. At this moment the resistance, the capacitance and the junction width start to level off indicating the convergence to a quasi steady state. After $250 \mathrm{~h}$ of driving, the junction thickness has decreased marginally onwards to $58 \mathrm{~nm}$ meaning that the crossover region from $\mathrm{p}$ - to n-type doping occupies most of the active layer. The large width of the intrinsic region at this point in time is responsible for the huge resistance of $3.6 \mathrm{k} \Omega$ resulting in a small current and moreover in a low probability of radiative charge carrier recombination. Both of these effects may rationalize the low device brightness of around $100 \mathrm{~cd} / \mathrm{m}^{2}$ at this instant of time (cf. Table III).

To briefly comment on the time-dependent luminescent behavior of the iTMC-LEC under bias, the onset of electroluminescence, defined as the moment where the luminance reached a value of $1 \mathrm{~cd} / \mathrm{m}^{2}$, was detected after $22 \mathrm{~h}$ of operation. At this point in time, the undoped part of the active layer spans roughly $80 \%$ of the total thickness giving rise to a p-i-n junction with a vast intrinsic area. Henceforth and within the timeframe of the experiment, the brightness increases continuously with proceeding driving time and follows the trend of the current. Particularly, when the capacitance and the resistance of the intrinsic region start to level off after around $100 \mathrm{~h}$ of operation and the junction width approaches a quasi steady state, the rise of the current and luminance is still ongoing. This observation is indicative of an increase in the doping density in the p- and/or n-doped zones in the advanced stage of operation.

So far, only the evolution of the intrinsic region during iTMC-LEC operation was discussed. In the following, we will finally comment on the doped zones and their development as a function of time. After $22 \mathrm{~h}$ of driving, when light emission is observed for the first time and in consequence, the p-i-n junction has formed, the resistances of the $\mathrm{p}$ - and ntype regions, as derived from the CNLS fit, are relatively high (lower $\mathrm{k} \Omega$ range) taking into account that the active layer has undergone doping. Moreover, they also differ quite distinctly ( 1 vs. $10 \mathrm{k} \Omega$ ). The first issue may be explained by a generally low doping density in the $\mathrm{p}$ - and n-type regions or by a gradual change in the doping density and hence conductivity throughout the doped zones as it was demonstrated to occur in pLECs. ${ }^{48}$ Recalling the discussion on a proper equivalent circuit to fit the IS data of the iTMC-LEC under bias, it is exactly the latter assertion that was adduced to justify the use of resistor/CPE assemblies instead of pure resistors to model the doped zones. In addition, the second finding implies a significant difference in the doping density among both regions, a fact that might bear upon the very different injection barriers for electrons $(1.6 \mathrm{eV})$ and holes $(0.2 \mathrm{eV})$ in the present device, that may at least partly persist even in the presence of doping. ${ }^{63}$ In view of the latter statement, the doped zone with the higher resistance should be ascribed ntype behavior, while the one with the lower resistance should be attributed p-type conductivity. With proceeding operating time, the resistance of the p-doped zone is not subjected to any significant changes which imply a virtually constant doping density throughout this region. In contrast, the resistance of the n-doped zone drops markedly indicating an increase in the doping density with time. This may explain the ongoing rise in current and luminance when the junction width has started to approach a quasi steady state. As a last point, it is to be noted that the fitting results can neither unequivocally confirm nor disprove a migration of the $\mathrm{p}-\mathrm{i}-\mathrm{n}$ junction during operation as postulated in the first part of this article.

Finally, it is worth mentioning that also early experiments on pLECs revealed that the width of the intrinsic region constitutes a large portion of the total device thickness at a low driving voltage of $3 \mathrm{~V}$ (approximately $80 \%) .^{42}$ Lately, van Reenen et al. demonstrated a junction width to active layer thickness ratio of $0.3-0.8$ dependent on the semiconducting polymer used. ${ }^{48}$ In case of an iTMC-LEC comprising an iridium(III) complex similar to the one used in this work, Lenes et al. recently highlighted a strong decrease in the effective thickness from $120 \mathrm{~nm}$ to $26 \mathrm{~nm}$ at a low operating voltage of $3.5 \mathrm{~V} .{ }^{38}$ In general, thinner junctions were observed at higher operating voltages ${ }^{47,52}$ and for devices with higher ionic conductivity, respectively. ${ }^{52}$

\section{CONCLUSION}

Impedance spectroscopy proves to be a powerful tool to shed light on the dynamic processes taking place in ionic transition metal complex-based LECs featuring a thin-film architecture. Supported by complex nonlinear least 
squares fitting technique, important key structural and kinetic data of devices comprising the ionic compound $\left[\operatorname{Ir}(\mathrm{ppy})_{2}(\mathrm{pbpy})\right]\left[\mathrm{PF}_{6}\right]$ could be derived. Measurements where the bias voltage did not exceed the bandgap potential of the complex used were applied to determine the electric double layer thickness and the conductivity and mobility, respectively, of the mobile anions. Moreover, we found that charge transport in the investigated devices is dominated by the ions when carrier injection from the electrodes is prohibited and that ion movement is in general limited by the presence of transverse internal interfaces. Measurements at bias voltages exceeding the bandgap potential gave rise to an increase in capacitance which was used to calculate the width of the intrinsic region during operation. We showed that the p-i-n junction constitutes almost $60 \%$ of the total active layer thickness in steady state at an operating voltage only slightly higher than the bandgap potential which is obviously the cause for the low current and luminance observed under these driving conditions.

\section{ACKNOWLEDGMENTS}

The authors thank Dr. G. Schmid and Dr. A. Kanitz from the Siemens AG for the supply of the ionic iridium(III) complex and Sabine Szyszkowski for the preparation of the devices. Financial support from the European Community's Seventh Framework Program under Grant Agreement No. P7-ICT-248043 (CELLO) is gratefully acknowledged.

${ }^{1}$ N. Holonyak, Jr. and S. F. Bevacqua, Appl. Phys. Lett. 1, 82 (1962).

${ }^{2}$ M. Shur and A. Žukauskas, Int. J. High Speed Electron. Syst. 20, 229 (2011).

${ }^{3}$ A. K. Viswanath, J. Nanosci. Nanotechnol. 14, 1947 (2014).

${ }^{4}$ Y.-S. Tyan, J. Photonics Energy 1, 011009 (2011).

${ }^{5}$ T. S. Perry, IEEE Spectrosc. 50, 46 (2013).

${ }^{6}$ S. Murano and A. Lemke, Inf. Disp. 29, 24 (2013).

${ }^{7}$ C. Nguyen, G. Ingram, and Z. Lu, Proc. SPIE 8983, 89830C (2014).

${ }^{8}$ H. Sasabe and J. Kido, J. Mater. Chem. C 1, 1699 (2013).

${ }^{9}$ Q. Pei, G. Yu, C. Zhang, Y. Yang, and A. J. Heeger, Science 269, 1086 (1995).

${ }^{10}$ Q. Pei, Y. Yang, G. Yu, C. Zhang, and A. J. Heeger, J. Am. Chem. Soc. 118, 3922 (1996).

${ }^{11}$ Q. Sun, Y. Li, and Q. Pei, J. Disp. Technol. 3, 211 (2007).

${ }^{12}$ Z. Yu, L. Li, H. Gao, and Q. Pei, Sci. China Chem. 56, 1075 (2013).

${ }^{13}$ J.-K. Lee, D. S. Yoo, E. S. Handy, and M. F. Rubner, Appl. Phys. Lett. 69, 1686 (1996).

${ }^{14}$ T. Hu, L. He, L. Duan, and Y. Qiu, J. Mater. Chem. 22, 4206 (2012).

${ }^{15}$ R. D. Costa, E. Ortí, H. J. Bolink, F. Monti, G. Accorsi, and N. Armaroli, Angew. Chem. Int. Ed. 51, 8178 (2012).

${ }^{16}$ J. C. deMello, N. Tessler, S. C. Graham, and R. H. Friend, Phys. Rev. B 57, 12951 (1998).

${ }^{17}$ J. C. deMello, J. J. M. Halls, S. C. Graham, N. Tessler, and R. H. Friend, Phys. Rev. Lett. 85, 421 (2000).

${ }^{18}$ J. C. deMello, Phys. Rev. B 66, 235210 (2002).

${ }^{19}$ J. D. Slinker, J. A. DeFranco, M. J. Jaquith, W. R. Silveira, Y.-W. Zhong, J. M. Moran-Mirabal, H. G. Craighead, H. D. Abruña, J. A. Marohn, and G. G. Malliaras, Nature Mater. 6, 894 (2007).

${ }^{20} \mathrm{P}$. Matyba, K. Maturova, M. Kemerink, N. D. Robinson, and L. Edman, Nature Mater. 8, 672 (2009).

${ }^{21}$ S. B. Meier, S. van Reenen, B. Lefevre, D. Hartmann, H. J. Bolink, A. Winnacker, W. Sarfert, and M. Kemerink, Adv. Funct. Mater. 23, 3531 (2013).

${ }^{22}$ P. Matyba, H. Yamaguchi, G. Eda, M. Chhowalla, L. Edman, and N. D. Robinson, ACS Nano 4, 637 (2010).

${ }^{23}$ P. Matyba, H. Yamaguchi, M. Chhowalla, N. D. Robinson, and L. Edman, ACS Nano 5, 574 (2011).

${ }^{24}$ J. C. deMello, Nature Mater. 6, 796 (2007).
${ }^{25}$ Q. Pei and A. J. Heeger, Nature Mater. 7, 167 (2008).

${ }^{26}$ G. G. Malliaras, J. D. Slinker, J. A. DeFranco, M. J. Jaquith, W. R. Silveira, Y.-W. Zhong, J. M. Moran-Mirabal, H. G. Craighead, H. D. Abruña, and J. A. Marohn, Nature Mater. 7, 168 (2008).

${ }^{27}$ S. van Reenen, P. Matyba, A. Dzwilewski, R. A. J. Janssen, L. Edman, and M. Kemerink, J. Am. Chem. Soc. 132, 13776 (2010).

${ }^{28}$ T. J. Mills and M. C. Lonergan, Phys. Rev. B 85, 035203 (2012).

${ }^{29}$ J. Gao and J. Dane, Appl. Phys Lett. 84, 2778 (2004).

${ }^{30}$ Y. Hu, C. Tracy, and J. Gao, Appl. Phys. Lett. 88, 123507 (2006).

${ }^{31}$ Y. Hu and J. Gao, J. Am. Chem. Soc. 133, 2227 (2011).

${ }^{32}$ S. van Reenen, P. Matyba, A. Dzwilewski, R. A. J. Janssen, L. Edman, and M. Kemerink, Adv. Funct. Mater. 21, 1795 (2011).

${ }^{33}$ A. Inayeh, B. Dorin, and J. Gao, Appl. Phys. Lett. 101, 253305 (2012).

${ }^{34}$ L. S. C. Pingree, D. B. Rodovsky, D. C. Coffey, G. P. Bartholomew, and D. S. Ginger, J. Am. Chem. Soc. 129, 15903 (2007).

${ }^{35}$ D. J. Dick, A. J. Heeger, Y. Yang, and Q. Pei, Adv. Mater. 8, 985 (1996).

${ }^{36}$ Y. Hu, B. Dorin, F. Teng, and J. Gao, Org. Electron. 13, 361 (2012).

${ }^{37}$ Y. Lei, F. Teng, Y. Hou, Z. Lou, and Y. Wang, Appl. Phys. Lett. 95, 101105 (2009).

${ }^{38}$ M. Lenes, G. Garcia-Belmonte, D. Tordera, A. Pertegás, J. Bisquert, and H. J. Bolink, Adv. Funct. Mater. 21, 1581 (2011).

${ }^{39}$ S. B. Meier, D. Hartmann, D. Tordera, H. J. Bolink, A. Winnacker, and W. Sarfert, Phys. Chem. Chem. Phys. 14, 10886 (2012).

${ }^{40}$ G. Gozzi, L. F. Santos, and R. M. Faria, Europhys. Lett. 100, 18001 (2012).

${ }^{41}$ I. H. Campbell, D. L. Smith, C. J. Neef, and J. P. Ferraris, Appl. Phys. Lett. 72, 2565 (1998).

${ }^{42}$ Y. Li, J. Gao, G. Yu, Y. Cao, and A. J. Heeger, Chem. Phys. Lett. 287, 83 (1998).

${ }^{43}$ Y. Li, J. Gao, D. Wang, G. Yu, Y. Cao, and A. J. Heeger, Synth. Met. 97, 191 (1998).

${ }^{44}$ G. Yu, Y. Cao, C. Zhang, Y. Li, J. Gao, and A. J. Heeger, Appl. Phys. Lett. 73, 111 (1998).

${ }^{45}$ Y. A. Davis, P. P. Crooker, N. M. Haegel, Y. Yoshioka, and J. D. MacKenzie, Appl. Phys. Lett. 99, 233306 (2011).

${ }^{46}$ G. Gozzi, R. M. Faria, and L. F. Santos, Appl. Phys. Lett. 101, 113305 (2012).

${ }^{47}$ A. Munar, A. Sandström, S. Tang, and L. Edman, Adv. Funct. Mater. 22, 1511 (2012).

${ }^{48}$ S. van Reenen, R. A. J. Janssen, and M. Kemerink, Adv. Funct. Mater. 22, 4547 (2012)

${ }^{49}$ H. Rudmann, S. Shimada, M. F. Rubner, D. W. Oblas, and J. E. Whitten, J. Appl. Phys. 92, 1576 (2002).

${ }^{50}$ G. Kalyuzhny, M. Buda, J. McNeill, P. Barbara, and A. J. Bard, J. Am. Chem. Soc. 125, 6272 (2003)

${ }^{51}$ H. Rudmann, S. Shimada, and M. F. Rubner, J. Am. Chem. Soc. 124, 4918 (2002).

${ }^{52}$ H. Rudmann, S. Shimada, and M. F. Rubner, J. Appl. Phys. 94, 115 (2003).

${ }^{53}$ S. van Reenen, T. Akatsuka, D. Tordera, M. Kemerink, and H. J. Bolink, J. Am. Chem. Soc. 135, 886 (2013).

${ }^{54}$ H. J. Bolink, E. Coronado, R. D. Costa, E. Ortí, M. Sessolo, S. Graber, K. Doyle, M. Neuburger, C. E. Housecroft, and E. C. Constable, Adv. Mater. 20, 3910 (2008).

${ }^{55}$ F. Gärtner, D. Cozzula, S. Losse, A. Boddien, G. Anilkumar, H. Junge, T. Schulz, N. Marquet, A. Spannenberg, S. Gladiali, and M. Beller, Chem. Eur. J. 17, 6998 (2011).

${ }^{56}$ S. T. Parker, J. D. Slinker, M. S. Lowry, M. P. Cox, S. Bernhard, and G. G. Malliaras, Chem. Mater. 17, 3187 (2005).

${ }^{57}$ G. J. Brug, A. L. G. van den Eeden, M. Sluyters-Rehbach, and J. H. Sluyters, J. Electroanal. Chem. Interfacial Electrochem. 176, 275 (1984).

${ }^{58}$ B. Hirschorn, M. E. Orazem, B. Tribollet, V. Vivier, I. Frateur, and M. Musiani, Electrochim. Acta 55, 6218 (2010).

${ }^{59} \mathrm{E}$. Barsoukov and J. R. Macdonald, Impedance Spectroscopy: Theory, Experiment, and Applications (John Wiley \& Sons, New York, 2005).

${ }^{60}$ The total capacitance of capacitors in series is equal to the reciprocal of the sum of the reciprocals of the individual capacitances. Accordingly, when assuming a similar capacitance of both EDLs, the capacitance of each single EDL is twice the value of the low-frequency capacitance plateau $(14.69 \mathrm{nF})$.

${ }^{61}$ R. A. Huggins, Ionics 8, 300 (2002).

${ }^{62}$ J. R. Macdonald, Ann. Biomed. Eng. 20, 289 (1992).

${ }^{63}$ C.-T. Liao, H.-F. Chen, H.-C. Su, and K.-T. Wong, Phys. Chem. Chem. Phys. 14, 9774 (2012). 